\title{
Turbo- and BCH-Coded Wide-Band Burst-by-Burst Adaptive H.263-Assisted Wireless Video Telephony
}

\author{
Peter Cherriman, Choong Hin Wong, and Lajos Hanzo
}

\begin{abstract}
The video performance benefits of burst-by-burst adaptive modulation are studied, employing a higher-order modulation scheme when the channel is favorable, in order to increase the system's bits per symbol capacity and conversely, invoking a more robust lower order modulation scheme when the channel exhibits inferior channel quality. It is shown that due to the proposed adaptive modem mode switching regime, a seamless video-quality versus channel quality relationship can be established, resulting in error-free video quality right across the operating channel signal-to-noise ratio (SNR) range. The main advantage of the proposed burst-by-burst adaptive transceiver technique is that irrespective of the prevailing channel conditions, the transceiver achieves always the best possible source-signal representation quality - such as video, speech, or audio quality-by automatically adjusting the achievable bitrate and the associated multimedia source-signal representation quality in order to match the channel quality experienced. This is achieved on a near-instantaneous basis under given propagation conditions in order to cater for the effects of path loss, fast-fading, slow-fading, dispersion, co-channel interference, etc. Furthermore, when the mobile is roaming in a hostile out-doors-or even hilly terrain-propagation environment, typically low-order low-rate modem modes are invoked, while in benign indoor environments, predominantly the high-rate high source-signal representation quality modes are employed.
\end{abstract}

Index Terms-AQAM, burst-by-burst adaptive modems, H.263, modulation, video telephony, wireless video.

\section{BACKGROUND}

$\mathbf{T}$ HE PHILOSOPHY of the fixed, but programable-rate, proprietary video codecs and statically reconfigurable ${ }^{1}$ multi-mode video transceivers presented by Streit et al. in

Manuscript received December 3, 1998; revised February 22, 2000. This work was supported by the Mobile Virtual Centre of Excellence, U.K., by EPSRC, Swindon, U.K., and by the European Commission. This paper was recommended by Associate Editor Y. Wang.

The authors are with the Department of Electronics and Computer Science, University of Southampton, Southampton SO17 1BJ, U.K. (e-mail pjc@ecs.soton.ac.uk; 1h@ecs.soton.ac.uk).

Publisher Item Identifier S 1051-8215(00)10626-3.

${ }^{1}$ In contrast to the burst-by-burst reconfigurable wideband video transceivers proposed in this contribution, the term statically reconfigurable in this context refers to video transceivers that cannot be near-instantaneously reconfigured. More explicitly, the previously proposed statically reconfigurable video transceivers were reconfigured on a longer term basis under the base station's control, invoking for example in the central cell region - where benign channel conditions prevail-a less robust, but high-throughput modulation mode, such as 4 bits/symbol quadrature amplitude modulation [1], [2] (16QAM), which was capable of transmitting a quadruple number of bits, and hence ensured a better video quality. By contrast, a robust, but low-throughput modulation mode, such as $1 \mathrm{bit} / \mathrm{symbol}$ binary phase shift keying (BPSK), can be employed near the edge of the propagation cell, where hostile propagation conditions prevail. This prevented a premature hand-over at the cost of a reduced video quality.
[2]-[6] was that irrespective of the video-motion activity experienced, the specially designed video codecs generated a constant number of bits per video frame. For example, for videophone over the second-generation Global System of Mobile Communications, known as the GSM system [7], at $13 \mathrm{kbits} / \mathrm{s}$ and assuming a video scanning rate of 10 frames/s, 1300 bits per video frame have to be generated. ${ }^{2}$ Specifically, two families of video codecs were designed, one refraining from using error-sensitive run-length coding techniques and exhibiting the highest possible error resilience, and another aiming for the highest possible compression ratio. This fixed-rate approach had the advantage of requiring no adaptive feedback controlled bitrate fluctuation smoothing buffering, and hence exhibited no objectionable video latency or delay. Furthermore, these video codecs were amenable to video telephone over fixed-rate second-generation mobile radio systems, such as the GSM [7].

The fixed bitrate of the above proprietary video codecs is in contrast to existing standard video codecs, such as the Motion Pictures Expert Group codecs known as MPEG1 [8] and MPEG2 [9], or the ITU's H.263 codec [10], where the time-variant video-motion activity and the variable-length coding techniques employed result in a time-variant bitrate fluctuation and a near-constant perceptual video quality. This time-variant bitrate fluctuation can be mitigated by employing adaptive feedback-controlled buffering, which potentially increases the latency or delay of the codec, and hence it is often objectionable in interactive videophone. The schemes presented by Streit et al. in [3]-[6] result in slightly variable video quality at a constant bitrate, while refraining from employing buffering, which again would result in latency in interactive videophone. A range of techniques, which can be invoked in order to render the family of variable-length coded highly bandwidth-efficient, but potentially error-sensitive, class of standard video codecs, such as the H.263 arrangement, amenable to error-resilient low-latency interactive wireless multimode videophone was summarized in [11] Of particular importance for this contribution will be the adaptive video-rate control and packetization algorithm of [11], which generates the required number of bits for the burst-by-burst adaptive transceiver, depending the on the capacity of the current packet, as determined by the current modem mode. Furthermore, error-resilient H.263-based schemes were contrived for example by Färber $e t$ al. at Erlangen University [12], while Sadka et al. [13] from Surrey University proposed a range of

\footnotetext{
${ }^{2} \mathrm{~A}$ range of video sequences encoded at various bitrates between 6.7 and 13 kbits/s can be viewed at http://www-mobile.ecs.soton.ac.uk, where the papers referenced can also be downloaded.
} 
improvements to the H.263 scheme. Following the above portrayal of the prior art in both video compression and statically reconfigurable narrowband modulation, let us now consider the philosophy of burst-by-burst adaptive wideband modulation in more depth.

In burst-by-burst adaptive modulation, a higher order modulation scheme is invoked when the channel is favorable, in order to increase the system's bits per symbol capacity and conversely, a more robust lower order modulation scheme is employed when the channel exhibits inferior channel quality, in order to improve the mean bit error ratio (BER) performance. A practical scenario, where adaptive modulation can be applied, occurs when a reliable low-delay feedback path is created between the transmitter and receiver, for example by superimposing the estimated channel quality perceived by the receiver on the reverse-direction messages of a duplex interactive channel. The transmitter then adjusts its modem mode according to this perceived channel quality.

The main advantage of the proposed burst-by-burst adaptive transceiver technique is that irrespective of the prevailing channel conditions, the transceiver achieves always the best possible source-signal representation quality — such as video, speech or audio quality - by automatically adjusting the achievable bitrate and the associated multimedia source-signal representation quality in order to match the channel quality experienced. This is achieved on a near-instantaneous basis under given propagation conditions in order to cater for the effects of path loss, fast-fading, slow-fading, dispersion, co-channel interference, etc. Furthermore, when the mobile is roaming in a hostile outdoor - or even hilly terrain-propagation environment, typically low-order low-rate modem modes are invoked, while in benign indoor environments, predominantly the high-rate high source-signal representation quality modes are employed.

Recent developments in adaptive modulation over a narrow-band channel environment have been pioneered by Webb and Steele [14], where the modulation adaptation was utilized in a Digital European Cordless telephone (DECT)-like system. The concept of variable rate adaptive modulation was then further developed by Sampei et al. [15], showing promising advantages when compared to fixed modulation in terms of spectral efficiency, BER performance, and robustness against channel delay spread. In another paper, the numerical upper bound performance of adaptive modulation in a slow Rayleigh flat-fading channel was evaluated by Torrance $e t$ al. [16] and subsequently, the optimization of the switching threshold levels using Powell minimization was proposed in order to achieve a targeted performance [17]. In addition, adaptive modulation was also studied in conjunction with channel coding and power control techniques by Matsuoka $e t$ al. [18], as well by as Goldsmith and Chua [19], [20].

In the narrow-band channel environment, the quality of the channel was determined by the short-term SNR of the received burst, which was then used as a criterion in order to choose the appropriate modulation mode for the transmitter, based on a list of switching threshold levels $l_{n}$ [14], [15], [16], [21]. However, in a wideband environment, this criterion is not an accurate measure for judging the quality of the channel, where the exis- tence of multi-path components produces not only power attenuation of the transmission burst, but also intersymbol interference. Subsequently, a new criterion has to be defined to estimate the wideband channel quality in order to choose the appropriate modulation scheme.

In addition, the wideband channel-induced degradation is combated not only by the employment of adaptive modulation but also by equalization. In following this line of thought, we can formulate a two-step methodology in mitigating the effects of the dispersive wideband channel. In the first step, the equalization process will eliminate most of the intersymbol interference based on a channel impulse response (CIR) estimate derived using the channel sounding midamble and consequently, the signal to noise and residual interference ratio at the output of the equalizer is calculated.

We found that the residual channel-induced inter-symbol-interference (ISI) at the output of the decision feedback equalizer (DFE) is near-Gaussian distributed and that if there are no decision feedback errors, the pseudo-SNR at the output of the DFE, $\gamma_{d f e}$ can be calculated as [25]

$$
\begin{aligned}
\gamma_{d f e} & =\frac{\text { Wanted Signal Power }}{E\left[\left|S_{k} \sum_{m=0}^{N_{f}-1} C_{m} h_{m}\right|^{2}\right]} \\
& =\frac{\sum_{q=-\left(N_{f}-1\right)}^{-1} E\left[\left|f_{q} S_{k-q}\right|^{2}\right]+N_{o} \sum_{m=0}^{N_{f}-1}\left|C_{m}\right|^{2}}{}
\end{aligned}
$$

where $C_{m}$ and $h_{m}$ denotes the DFE's feedforward coefficients and the channel impulse response, respectively. The transmitted signal and the noise spectral density is represented by $S_{k}$ and $N_{o}$. Lastly, the number of DFE feedforward coefficients is denoted by $N_{f}$. By utilizing the pseudo-SNR at the output of the equalizer, we are ensuring that the system performance is optimized by employing equalization and adaptive quadrature amplitude modulation (AQAM) [1] in a wideband environment according to the following switching regime:

$$
\text { Modulation Mode }= \begin{cases}\text { BPSK, } & \text { if } \gamma_{\mathrm{DFE}}<f_{1} \\ \text { 4QAM, } & \text { if } f_{1}<\gamma_{\mathrm{DFE}}<f_{2} \\ \text { 16QAM, } & \text { if } f_{2}<\gamma_{\mathrm{DFE}}<f_{3} \\ 64 \mathrm{QAM}, & \text { if } \gamma_{D F E}>f_{3}\end{cases}
$$

where $f_{n}, n=1 \ldots 3$ are the pseudo-SNR thresholds levels, which are set according to the system's integrity requirements.

In summary, in contrast to the narrow-band statically reconfigured multimode systems of [3]-[6] in this treatise, we invoked wideband near-instantaneously reconfigured or burst-by-burst adaptive modulation in order to quantify the video performance benefits of such systems. It is an important element of the system that when the $\mathrm{BCH}$ codes protecting the video stream are overwhelmed by the plethora of transmission errors, we refrain from decoding the video packet in order to prevent error propagation through the reconstructed frame buffer [11]. Instead, these cor- 
TABLE I

MOdUlation AND CHANNEl PARAMETERS

\begin{tabular}{l|c}
\hline Parameter & Value \\
\hline \hline Carrier Frequency & $1.9 \mathrm{GHz}$ \\
\hline Vehicular Speed & $30 \mathrm{mph}$ \\
\hline Doppler frequency & $85 \mathrm{~Hz}$ \\
\hline Normalised Doppler fr. & $3.27 \times 10^{-5}$ \\
\hline Channel type & COST 207 Typ. Urban (Figure 1) \\
\hline No. of paths in channel & 4 \\
\hline Data modulation & Adaptive QAM \\
& (BPSK, 4-QAM, 16-QAM, 64-QAM) \\
\hline Receiver type & $\begin{array}{c}\text { Decision Feedback Equalizer } \\
\text { Number of Forward Filter Taps }=35 \\
\text { Number of Backward Filter Taps }=7\end{array}$ \\
\hline
\end{tabular}

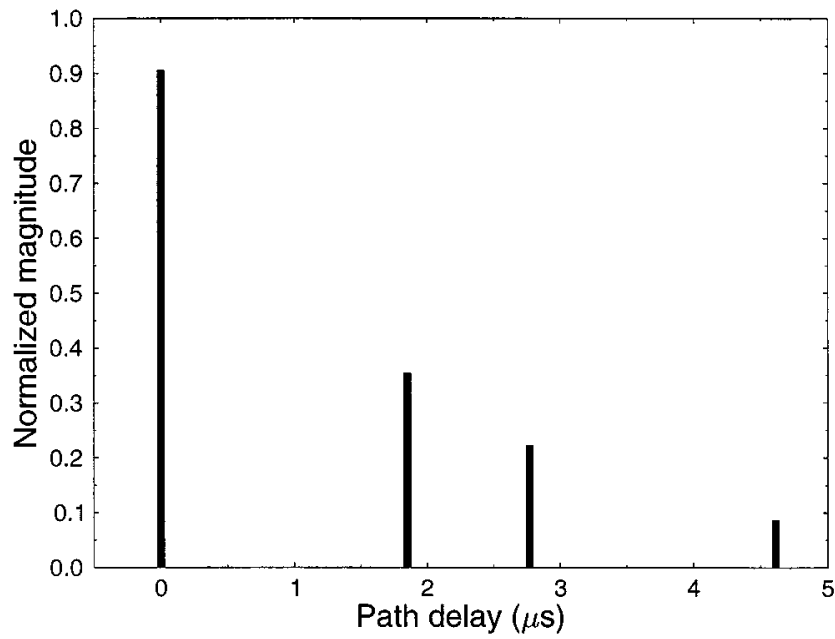

Fig. 1. Normalized channel impulse response for the COST 207 [25] four-path Typical Urban channel.

rupted packets are dropped and the reconstructed frame buffer will not be updated, until the next packet replenishing the specific video frame area arrives. The associated video performance degradation is fairly minor for packet dropping or frame error rates (FERs) below about 5\%. These packet dropping events are signaled to the remote decoder by superimposing a strongly protected 1-bit packet acknowledgment flag on the reverse-direction packet, as outlined in [11]. In the proposed scheme we also invoked the adaptive rate control and packetization algorithm of [11], supporting constant baud-rate operation.

The paper is structured as follows. Section II introduces the video transceiver parameters, while the bulk of the paper is constituted by the video performance analysis of Section III. Section IV characterizes the effects of adaptive modem mode switching thresholds on the system's video performance, while Section V characterizes the turbo-coded system's performance, before concluding in Section VI.

\section{VIDEO TRANSCEIVER}

In this contribution we used $176 \times 144$ pixel QCIF-resolution, 30 frames/s video sequences encoded at bitrates resulting in high perceptual video quality. Table I shows the modulation- and channel parameters employed. The COST207 [26] four-path typical urban (TU) channel model was used and its

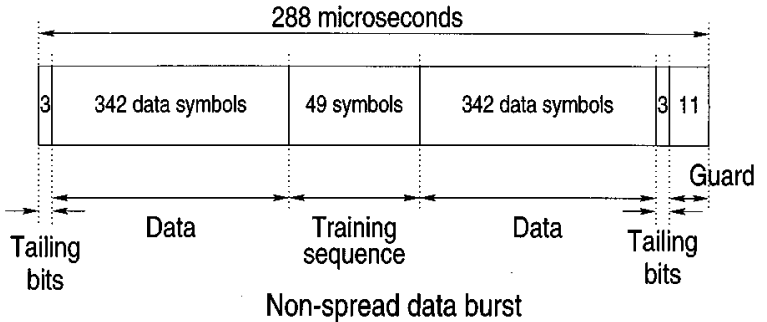

Fig. 2. Transmission burst structure of the FMA1 nonspread data burst mode of the FRAMES proposal [27].

TABLE II

Generic System FeAtures of the ReCONFIGURABle Multi-Mode VideO TRANSCEIVER, USING THE NON-SPREAD DATA BURST MODE OF THE FRAMES PROPOSAL [27] SHOWN IN FIG. 2

\begin{tabular}{l||c}
\hline Features & Value \\
\hline \hline Multiple access & TDMA \\
\hline No. of Slots/Frame & 16 \\
\hline TDMA frame length & $4.615 \mathrm{~ms}$ \\
\hline TDMA slot length & $288 \mu \mathrm{s}$ \\
\hline Data Symbols/TDMA slot & 684 \\
\hline User Data Symbol Rate (KBd) & 148.2 \\
\hline System Data Symbol Rate (MBd) & 2.37 \\
\hline Symbols/TDMA slot & 750 \\
\hline User Symbol Rate (KBd) & 162.5 \\
\hline System Symbol Rate (MBd) & 2.6 \\
\hline System Bandwidth (MHz) & 3.9 \\
\hline Eff. User Bandwidth $(\mathrm{kHz})$ & 244 \\
\hline
\end{tabular}

impulse response is portrayed in Fig. 1. We used the Pan-European FRAMES proposal [27] as the basis for our wideband transmission system, the frame structure of which is shown in Fig. 2. Employing the FRAMES Mode A1 (FMA1) so-called nonspread data burst mode required a system bandwidth of 3.9 $\mathrm{MHz}$, when assuming a modulation excess bandwidth of $50 \%$. A range of other system parameters are shown in Table II.

The proposed video transceiver is based on the H.263 video codec [10]. The video coded bitstream was protected by binary Bose-Chaudhuri-Hocquenghem $(\mathrm{BCH})$ coding [7] combined with an intelligent burst-by-burst adaptive wideband multi-mode quadrature amplitude modulation (QAM) modem [1], which can be configured either under network control or under transceiver control to operate as a 1,2, 4, and 6 bits/symbol scheme, while maintaining a constant signaling rate. This allowed us to support an increased throughput expressed in terms of the average number of bits per symbol, when the instantaneous channel quality was high, leading ultimately to an increased video quality in a constant bandwidth.

The transmitted bitrate for all four modes of operation is shown in Table III. The unprotected bitrate before approximately half-rate $\mathrm{BCH}$ coding is also shown in the table. The actual useful bitrate available for video is slightly less, than the unprotected bitrate due to the required strongly protected packet acknowledgment information and packetization information. The effective video bitrate is also shown in the Table III.

\section{BURST-By-Burst AdAPTIVE VIDEOPHONE PERFORMANCE}

The proposed burst-by-burst adaptive modem maximizes the system capacity available by using the most appropriate mod- 
TABLE III

OPERATIONAL-Mode SPECIFIC TRANSCEIVER PARAMETERS

\begin{tabular}{l||c|c|c|c}
\hline \multicolumn{1}{l||}{} & \multicolumn{4}{c}{ Multi-rate System } \\
\hline \hline Meatures & BPSK & 4QAM & 16QAM & 64 QAM \\
\hline Bits/Symbol & 1 & 2 & 4 & 6 \\
\hline FEC & \multicolumn{4}{c}{ Near Half-rate BCH } \\
\hline $\begin{array}{l}\text { Transmission } \\
\text { bitrate (kbit/s) }\end{array}$ & 148.2 & 296.4 & 592.8 & 889.3 \\
\hline $\begin{array}{l}\text { Unprotected } \\
\text { bitrate (kbit/s) }\end{array}$ & 75.8 & 151.7 & 303.4 & 456.1 \\
\hline $\begin{array}{l}\text { Effective } \\
\text { Video-rate (kbit/s) }\end{array}$ & 67.0 & 141.7 & 292.1 & 446.4 \\
\hline Video fr. rate (Hz) & \multicolumn{5}{|c}{30} \\
\hline
\end{tabular}

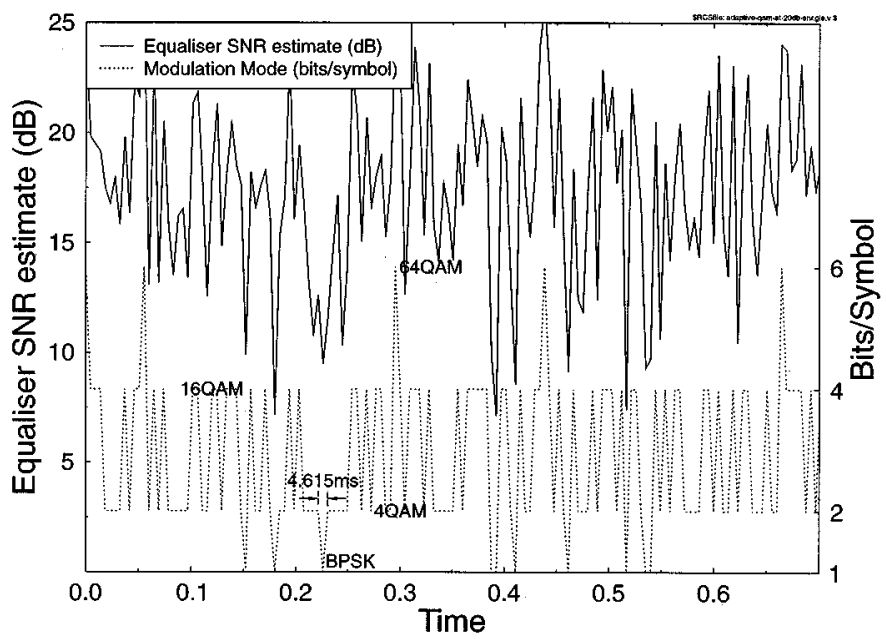

Fig. 3. Adaptive burst-by-burst modem in operation for an average channel SNR of $20 \mathrm{~dB}$, where the modulation node switching is based upon the SNR estimate at the output of the equalizer using the channel parameters defined in Table I.

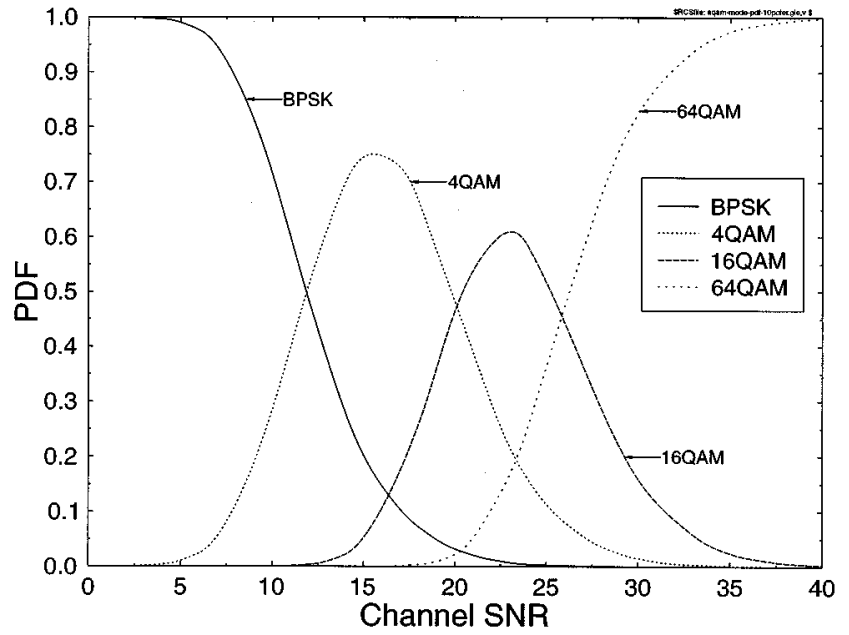

Fig. 4. PDF of the adaptive modem being in a particular modulation mode versus channel SNR.

ulation mode for the current instantaneous channel conditions. We found that the pseudo-SNR at the output of the channel equalizer was an adequate channel quality measure in our burst-by-burst adaptive wide-band modem. Fig. 3 demonstrates how the burst-by-burst adaptive modem changes its modulation modes every transmission burst, i.e., every $4.615 \mathrm{~ms}$, based on

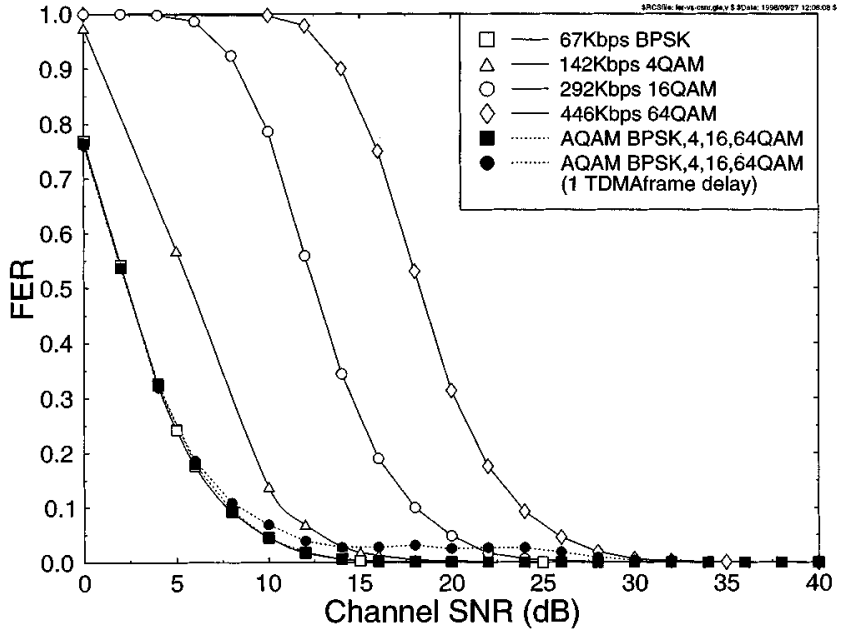

Fig. 5. Transmission FER (or packet loss ratio) versus channel SNR comparison of the four fixed-modulation modes (BPSK, 4QAM, 16QAM, 64QAM) and that of the adaptive burst-by-burst modem (AQAM). AQAM is shown with a realistic one TDMA frame delay between channel estimation and mode switching, and also with a zero delay version for indicating the upper bound performance. The channel parameters were defined in Table I.

the fluctuating pseudo-SNR. The right-hand-side vertical axis indicates the associated number of bits per symbol.

By changing to more robust modulation schemes automatically when the channel quality is reduced allows the packet loss ratio, or synonymously, the FER to be reduced, which results in increased perceived video quality. In order to judge the benefits of burst-by-burst adaptive modulation, we considered two scenarios. In the first scheme, the adaptive modem always chose the perfectly estimated AQAM modulation mode, in order to provide a maximum upper bound performance. In the second scenario, the modulation mode was based upon the perfectly estimated AQAM modulation mode for the previous burst, which corresponded to a delay of one time division multiple access (TDMA) frame duration of $4.615 \mathrm{~ms}$. This second scenario represents a practical burst-by-burst adaptive modem, where the one-frame channel quality estimation latency is due to superimposing the receiver's perceived channel quality on a reverse-direction packet for informing the transmitter concerning the best mode to be used.

The probability of the adaptive modem using each modulation mode for a particular average channel SNR is portrayed in Fig. 4 in terms of the associated modem mode probability density functions (PDFs). It can be seen at high channel SNRs that the modem mainly uses the 64QAM modulation mode, while at low channel SNRs the BPSK mode is the most prevalent one.

Fig. 5 shows the transmission FER (or packet loss ratio) versus channel SNR for the 1, 2, 4, and 6 bit/symbol fixed modulation schemes, as well as for the ideal and for the one-frame delayed realistic scenarios using the burst-by-burst adaptive QAM (AQAM) modem. A somewhat surprising fact is [28] - which is not explicitly shown here due to lack of space, that at low SNRs, AQAM can maintain a lower BER than BPSK, since under inferior instantaneous channel conditions it exhibits the corresponding BPSK BER, but the average number of transmitted AQAM bits is higher than that of BPSK, resulting in a reduced average AQAM BER. At high 
SNRs the AQAM BER curve asymptotically joins the 64QAM BER curve, since 64QAM is the predominant mode used. The associated FER curve obeys similar tendencies in terms of having the BPSK and 64QAM FER curves as asymptotes at low and high SNRs, respectively. However-in contrast to the BER - the AQAM FER cannot be lower than that of BPSK, since the same number of frames is transmitted in both cases. The substantial advantage of AQAM is that due to its higher number of bits/symbol the number bits transmitted per frame is higher, resulting in an increased video quality. ${ }^{3}$ The FER curves are portrayed on a logarithmic scale in Fig. 6 where-for the sake of comparison-we also showed the associated FER curve for statically reconfigured modem modes switching at 5\% transmission FER, as will be detailed below.

Explicitly, the statically reconfigured modem was invoked in Fig. 6 as a benchmarker, in order to indicate how a system would perform, which cannot act on the basis of the near-instantaneously varying channel quality. As can be inferred from Fig. 6, such a statically reconfigured transceiver switches its mode of operation from a lower order modem mode, e.g., BPSK, to a higher-order mode, e.g., 4QAM, when the channel quality has improved sufficiently for the 4QAM mode's FER to become lower than $5 \%$ upon reconfiguring the transceiver in this 4QAM mode. Again — as seen also in Fig. 5 earlier on a nonlogarithmic scale-Fig. 6 clearly shows on a logarithmic scale that the "one-frame channel estimation delay" AQAM modem manages to maintain a similar FER performance to the fixed-rate BPSK modem at low SNRs, although we will see in Fig. 7 that AQAM provides increasingly higher bitrates, reaching four times higher values than BPSK for high channel SNRs, where the employment of 64QAM is predominant. In this high SNR region the FER curve asymptotically approaches the 64QAM FER curve for both the realistic and the ideal AQAM scheme, although this is not visible in the figure for the ideal scheme, since this occurs at SNRs outside the range of Fig. 6. Again, the reason for this performance discrepancy is the occasionally misjudged channel quality estimates of the realistic AQAM scheme. Additionally, Fig. 6 indicates that the realistic AQAM modem exhibits a near-constant 3\% FER at medium SNRs. The issue of adjusting the switching thresholds in order to achieve the target FER will be addressed in detail in Section IV and the thresholds invoked will be detailed with reference to Table IV. Suffice to say at this stage that the average number of bits per symbol-and potentially also the associated video quality — can be increased upon using more aggressive switching thresholds. However, this results in an increased FER, which tends to decrease the video quality, as it will be discussed in Section IV.

A comparison of the effective video bitrate versus channel SNR is shown in Fig. 7 for the four fixed modulation schemes, and the ideal and realistic AQAM modems. The effective video bitrate is the average bitrate provided by all the successfully transmitted video packets. It should be noted that the realistic AQAM modem has a slightly lower throughput than the ideal, since occasionally the incorrect modulation mode is chosen,

\footnotetext{
${ }^{3}$ We note here that the associated performance results typically degrade upon increasing the Doppler frequency and improve upon reducing it, since the effects of channel estimation latency become less significant. This phenomenon was quantified in [28].
}

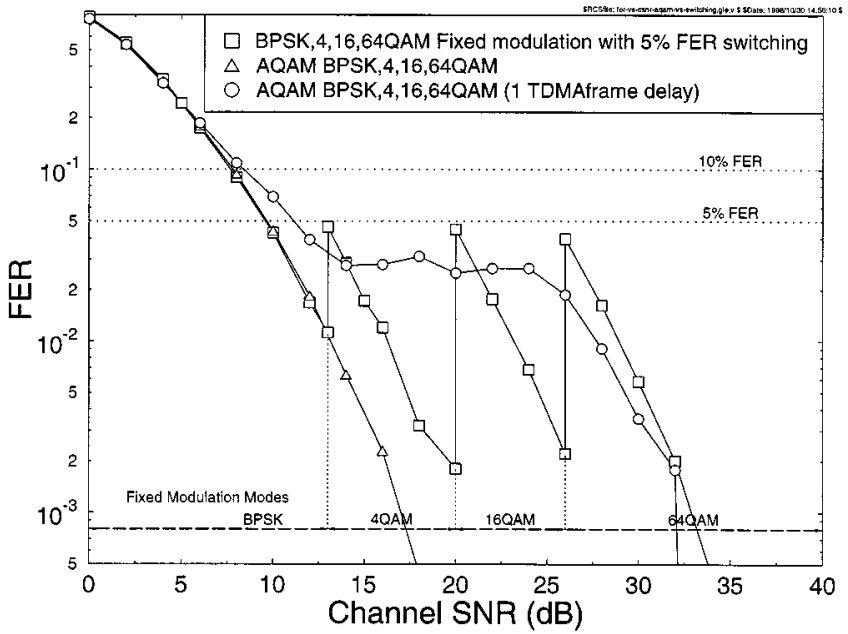

Fig. 6. Transmission FER (or packet loss ratio) versus channel SNR comparison of the four fixed modulation modes (BPSK, 4QAM, 16QAM, 64QAM) with 5\% FER switching and adaptive burst-by-burst modem (AQAM). AQAM is shown with a realistic one TDMA frame delay between channel estimation and mode switching, and a zero delay version is included as an upper bound. The channel parameters were defined in Table I.

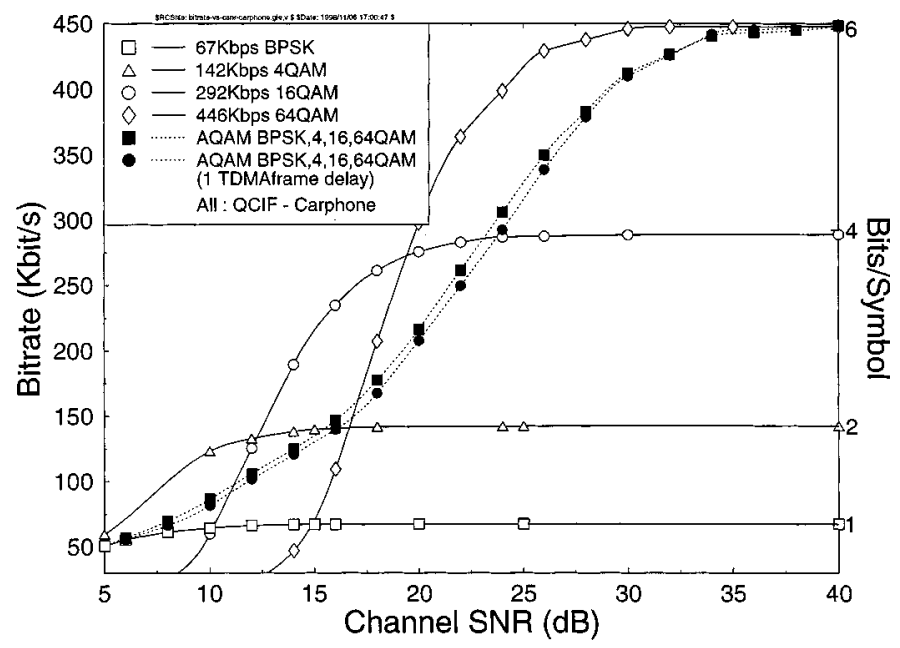

Fig. 7. Video bitrate versus channel SNR comparison of the four fixed modulation modes (BPSK, 4QAM, 16QAM, 64QAM) and adaptive burst-by-burst modem (AQAM). AQAM is shown with a realistic one TDMA frame delay between channel estimation and mode switching, and also as a zero delay version for indicating the upper bound. The channel parameters were defined in Table I.

TABLE IV

SINR ESTIMATE AT OUTPUT OF THE EQUALIZER REQUIRED FOR EACH MOdULATION MOdE IN BURST-BY-BURST ADAPTIVE MODEM. I.E., SWITCHING THRESHOLDS

\begin{tabular}{l|l|l|l|l}
\hline & BPSK & $4 \mathrm{QAM}$ & $16 \mathrm{QAM}$ & $64 \mathrm{QAM}$ \\
\hline \hline Standard & $<10 \mathrm{~dB}$ & $\geq 10 \mathrm{~dB}$ & $\geq 18 \mathrm{~dB}$ & $\geq 24 \mathrm{~dB}$ \\
\hline Conservative & $<13 \mathrm{~dB}$ & $\geq 13 \mathrm{~dB}$ & $\geq 20 \mathrm{~dB}$ & $\geq 26 \mathrm{~dB}$ \\
\hline Aggressive & $<9 \mathrm{~dB}$ & $\geq 9 \mathrm{~dB}$ & $\geq 17 \mathrm{~dB}$ & $\geq 23 \mathrm{~dB}$ \\
\hline
\end{tabular}

which may result in packet loss. At very low channel SNRs, the throughput bitrate converges to that of the fixed BPSK mode, since the AQAM modem is almost always in the BPSK mode at these SNRs, as demonstrated in Fig. 4. 
Having shown the effect of the burst-by-burst adaptive modem on the transmission FER and effective bitrate, let us now demonstrate these effects on the decoded video quality, measured in terms of the peak SNR (PSNR). Fig. 8 shows the decoded video quality in terms of PSNR versus channel SNR for both the ideal and realistic adaptive modem, and for the four modes of the statically configured multi-mode modem. It can be seen that-as expected - the ideal adaptive modem, which always selects the perfect modulation modes, has a better or similar video quality for the whole range of channel SNRs. For the statically configured multi-mode scheme the video quality degrades, when the system switches from a higher order to a lower order modulation mode. The ideal adaptive modem, however, smooths out the sudden loss of video quality, as the channel degrades. The nonideal adaptive modem has a slightly lower video quality performance, than the ideal adaptive modem, especially at medium SNRs, since it sometimes selects the incorrect modulation mode due to the estimation delay. This can inflict video packet loss and/or a reduction of the effective video bitrate, which in turn reduces the video quality.

The difference between the ideal burst-by-adaptive modem, using ideal channel estimation and the nonideal, realistic burst-by-burst adaptive modem, employing a nonideal delayed channel estimation can be seen more clearly in Fig. 9 for a range of video sequences. Observe that at high and low channel SNRs, the video quality performance is similar for the ideal and nonideal adaptive modems. This is because the channel estimation delay has little effect, since at low or high channel SNRs, the adaptive modem is in either BPSK or 64QAM mode for the majority of the time. More explicitly, the channel quality of a transmission frame is typically the same as that of the next, and hence the delay has little effect at low and high SNRs.

\section{SWITCHING THRESHOLDS}

The burst-by-burst adaptive modem changes its modulation modes based on the fluctuating channel conditions expressed in terms of the SNR at the equalizer's output. The set of switching thresholds used in all the previous graphs is the "Standard" set shown in Table IV, which was determined on the basis of the required channel SINR for maintaining the specific target video FER.

In order to investigate the effect of different sets of switching thresholds, we defined two new sets of thresholds: a more conservative set and a more aggressive set, employing less robust, but more bandwidth-efficient modem modes at lower SNRs. The more conservative switching thresholds reduced the transmission FER at the expense of a lower effective video bitrate. The more aggressive set of thresholds increased the effective video bitrate at the expense of a higher transmission FER.

The transmission FER performance of the realistic burst-byburst adaptive modem, which has a one TDMA frame delay between channel quality estimation and mode switching is shown in Fig. 10 for the three sets of switching thresholds of Table IV. It can be seen that the more conservative switching thresholds reduce the transmission FER from about 3\% to about $1 \%$ for medium channel SNRs. The more aggressive switching thresholds increase the transmission FER from about 3\% to $4-5 \%$.

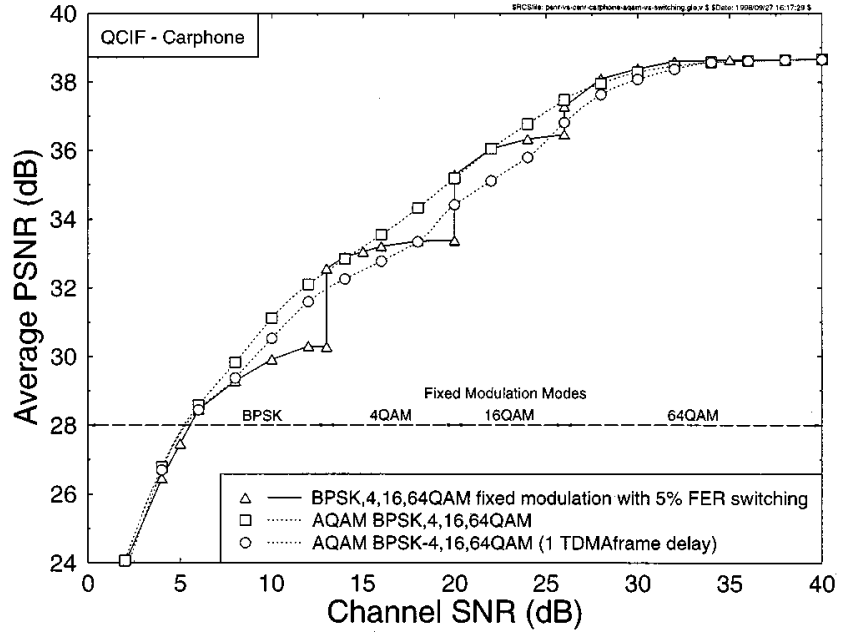

Fig. 8. Decoded video quality (PSNR) versus channel SNR comparison of the four fixed modulation modes (BPSK, 4QAM, 16QAM, 64QAM) with 5\% transmission FER switching and that of the adaptive burst-by-burst modem (AQAM). AQAM is shown with a realistic one TDMA frame delay between channel estimation and mode switching, and a zero delay version for indicating the upper bound. The channel parameters were defined in Table I.

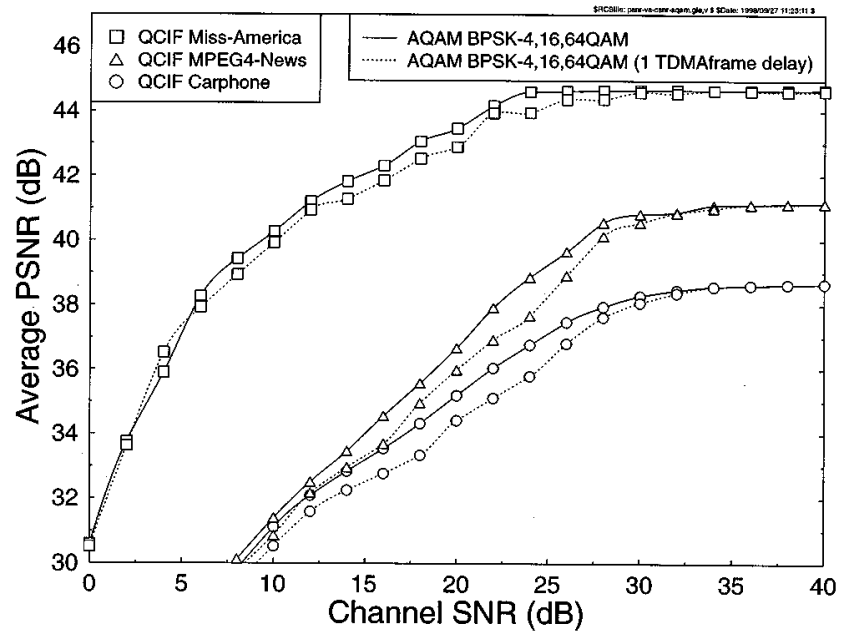

Fig. 9. Decoded video quality (PSNR) versus channel SNR for the adaptive burst-by-burst modem (AQAM). AQAM is shown with a realistic one TDMA frame delay between channel estimation and mode switching, and a zero delay version indicating the upper bound. Results are shown for three video sequences using the channel parameters that were defined in Table I.

However, since FERs below 5\% are not objectionable in video quality terms, this FER increase is an acceptable compromise for a higher effective video bitrate. The effective video bitrate for the realistic adaptive modem with the three sets of switching thresholds is shown in Fig. 11. The more conservative set of switching thresholds reduces the effective video bitrate but also reduces the transmission FER. The aggressive switching thresholds, increase the effective video bitrate, but also increase the transmission FER. Therefore the optimal switching thresholds should be set such that the transmission FER is deemed acceptable in the range of channel SNRs considered.

We note that the switching thresholds can be adjusted for example using Powell's optimization for uncoded transmissions [17], in order to achieve the required target BER and target bit rate upon defining an appropriately weighted joint cost function. 


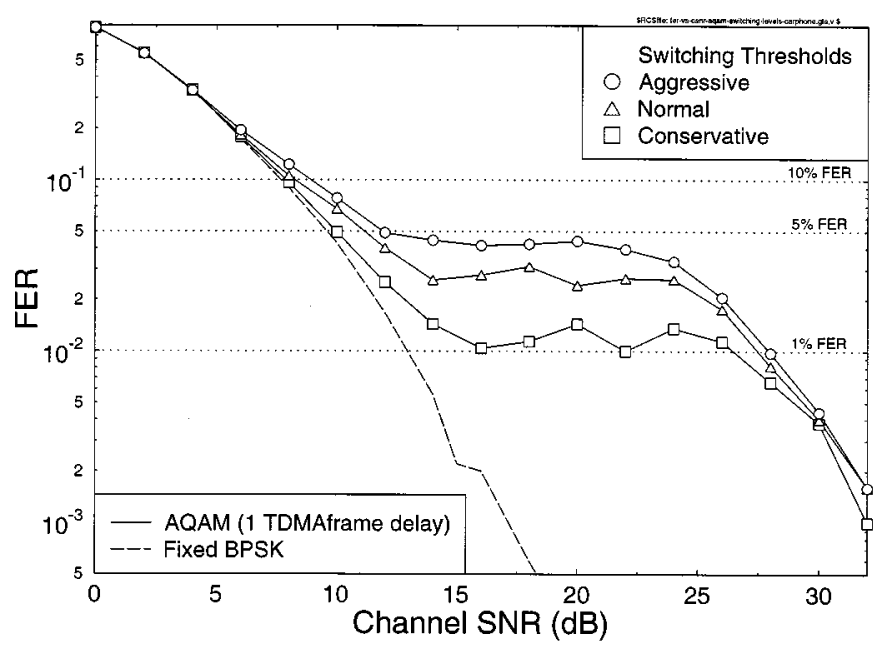

Fig. 10. Transmission FER (or packet loss ratio) versus channel SNR comparison of the fixed BPSK modulation mode and the adaptive burst-by-burst modem (AQAM) for the three sets of switching thresholds described in Table IV. AQAM is shown with a realistic one TDMA frame delay between channel estimation and mode switching. The channel parameters were defined in Table I.

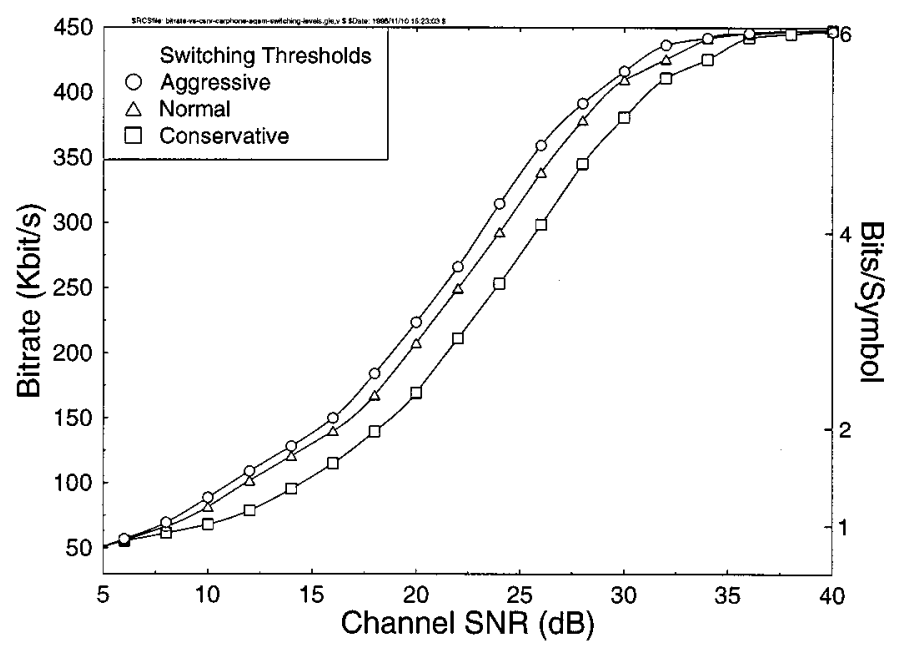

Fig. 11. Video bitrate versus channel SNR comparison for the adaptive burst-by-burst modem (AQAM) with a realistic one TDMA frame delay between channel estimation and mode switching for the three sets of switching thresholds as described in Table IV. The channel parameters were defined in Table I.

However, the coded AQAM system's BER/FER is not analytically tractable, and hence, no closed-loop optimization was invoked - the thresholds were adjusted experimentally. We note furthermore that instead of the equalizer's pseudo-SNR we also investigated using the channel codec's BER estimates for controlling the switching process, which resulted in a similar system performance. Let us now consider the performance improvements achievable when employing powerful turbo codecs.

\section{Turbo-Coded Video Performance}

In this section, we demonstrate the additional performance gains that are achievable, when turbo coding is used in preference to $\mathrm{BCH}$ coding. The generic system parameters of the turbo-coded reconfigurable multi-mode video transceiver are the same as those used in the $\mathrm{BCH}$-coded version summarized
TABLE $\mathrm{V}$

OPERATIONAL-Mode SPECIFIC TURBO-CODED TRANSCEIVER PARAMETERS

\begin{tabular}{l||c|c|c|c}
\hline \multicolumn{1}{l||}{ Features } & \multicolumn{4}{c}{ Multi-rate System } \\
\hline \hline Mode & BPSK & 4QAM & 16QAM & 64QAM \\
\hline Bits/Symbol & 1 & 2 & 4 & 6 \\
\hline FEC & \multicolumn{1}{c|}{ Half-Rate Turbo coding with CRC } \\
\hline $\begin{array}{l}\text { Transmission } \\
\text { bitrate (kbit/s) }\end{array}$ & 140.4 & 280.8 & 561.6 & 842.5 \\
\hline $\begin{array}{l}\text { Unprotected } \\
\text { bitrate (kbit/s) }\end{array}$ & 66.3 & 136.1 & 275.6 & 415.2 \\
\hline $\begin{array}{l}\text { Effective } \\
\text { Video-rate (kbit/s) }\end{array}$ & 60.9 & 130.4 & 270.0 & 409.3 \\
\hline Video fr. rate (Hz) & \multicolumn{5}{|c}{30} \\
\hline
\end{tabular}

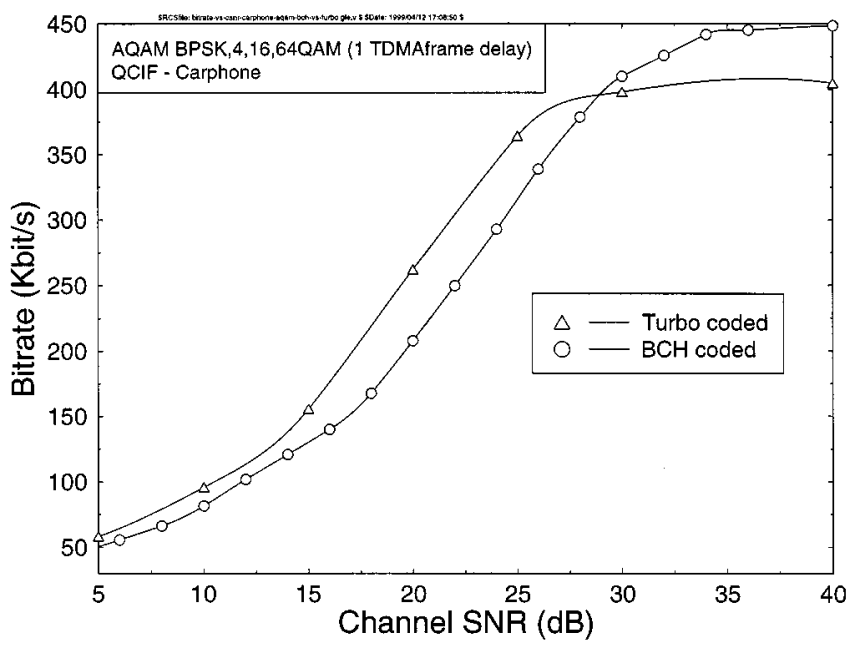

Fig. 12. Effective throughput video bitrate versus channel SNR comparison of the adaptive burst-by-burst modems (AQAM) using either $\mathrm{BCH}$ or turbo coding. The AQAM modems have a one TDMA frame delay between channel estimation and mode switching. The channel parameters were defined in Table I.

in Table II. Turbo-coding schemes are known to perform best in conjunction with square-shaped turbo interleaver arrays and their performance is improved upon extending the associated interleaving depth, since then the two constituent encoders are fed with more independent data. This ensures that the turbo decoder can rely on two quasiindependent data streams in its efforts to make as reliable decisions, as possible. A turbo interleaver size of $18 \times 18$ was chosen, requiring 324 bits for filling the interleaver. The required so-called recursive systematic convolutional component codes had a coding rate of $1 / 2$ and a constraint length of $K=3$. After channel coding the transmission burst length became 648 bits, which allowed the decoding of all AQAM transmission bursts independently. The operational-mode specific transceiver parameter are shown in Table V, and should be compared to the corresponding $\mathrm{BCH}$ coded parameters of Table III.

The turbo-coded parameters result in a $10 \%$ lower effective throughput bitrate compared to $\mathrm{BCH}$-coding under error-free conditions. However, we will show that under error-impaired conditions the turbo coding performance becomes better, resulting in a reduced video packet loss ratio. This reduced video packet loss ratio results in an increased effective throughput for a wide range of channel SNRs. Fig. 12 shows the effective throughput video bitrate versus channel SNR for the proposed AQAM modem using either $\mathrm{BCH}$ or turbo coding when the 


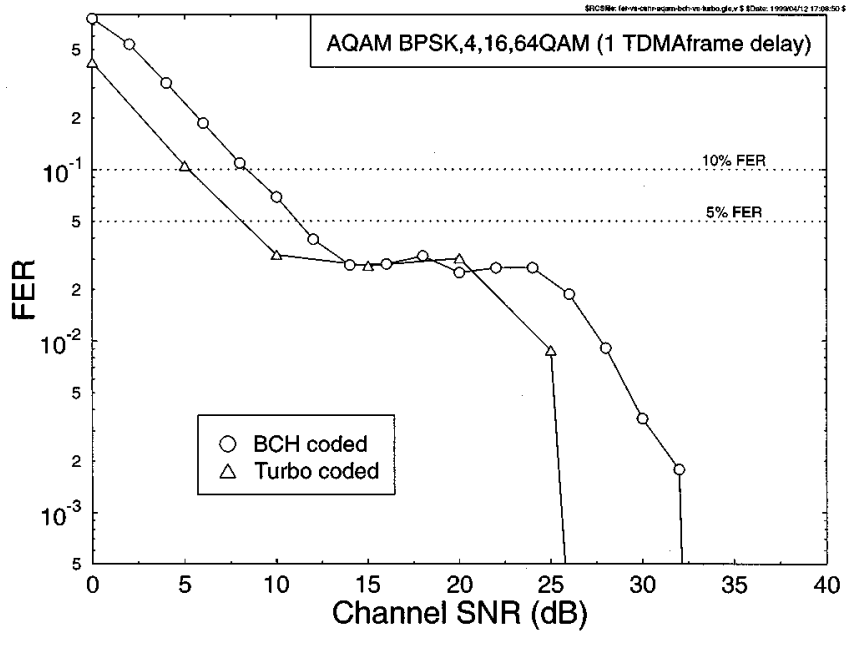

Fig. 13. Transmission FER (or packet loss ratio) versus channel SNR comparison of the adaptive burst-by-burst modem (AQAM) using the standard switching thresholds of Table IV, and either BCH or turbo coding. The channel parameters were defined in Table I and again, there was a one TDMA frame delay between channel estimation and mode switching.

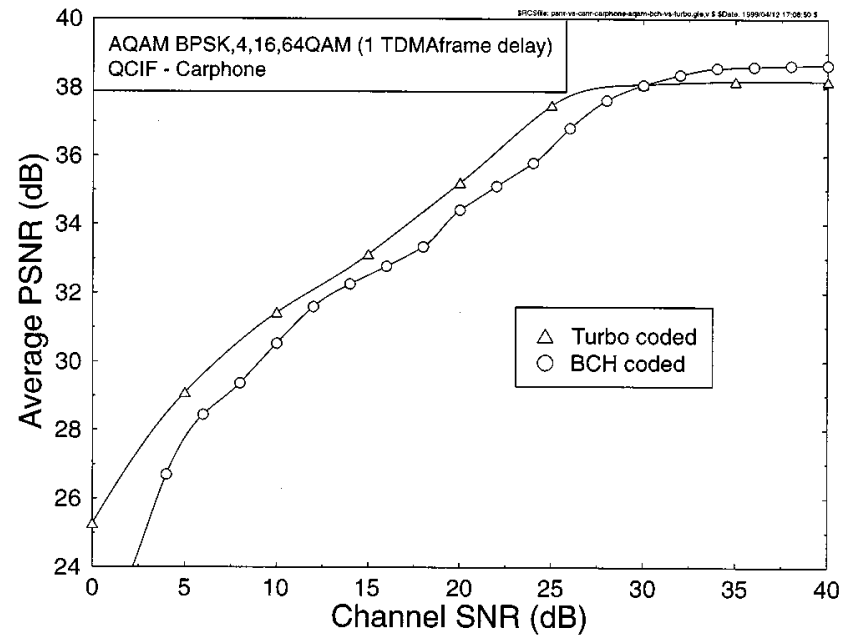

Fig. 14. Decoded video quality (PSNR) versus transmission FER (or packet loss ratio) comparison of the realistic adaptive burst-by-burst modems (AQAM) using either $\mathrm{BCH}$ or turbo coding. The channel parameters were defined in Table I.

delay between the channel quality estimate and mode switching of the AQAM modem was one TDMA frame duration.

At high channel SNRs and virtually error-free conditions the $\mathrm{BCH}$-coded modem slightly outperforms the turbo-coded modem in throughput bitrate terms, which were 446 and 409 kbits/ps respectively. However as the channel quality degrades, the lower packet loss ratio performance of the turbo-coded AQAM modem results in a higher effective throughput bitrate below channel SNRs of about $28 \mathrm{~dB}$. The associated FER or video packet loss ratio (PLR) performance versus channel SNR is shown in Fig. 13.

The BCH-coded and turbo-coded AQAM modems exhibit similarly shaped FER performance curves, both of which obey a similar FER performance curve to that of the corresponding 64QAM modem mode at high SNRs, whilst at low SNRs the FER curve follows that of the BPSK modem mode. For medium channel SNRs, the FER performance curve becomes near-constant, since the modem modes are adaptively adjusted, in order to maintain the required target FER. Observe, however, that the turbo-coded AQAM modem requires approximately a 3-dB lower channel SNR for achieving the required FER target. Hence, if the minimum acceptable video packet loss ratio is shown by the $5 \%$ limit drawn in the figure, the turbo-coded modem can maintain the required PLR for channel SNRs in excess of $8 \mathrm{~dB}$, while the $\mathrm{BCH}$-coded modem requires channel SNRs in excess of $11 \mathrm{~dB}$.

Having shown that the turbo-coded AQAM modem outperforms the $\mathrm{BCH}$ coded modem in terms of video packet loss performance, which results in a higher effective throughput video bitrate for all except very high channel SNRs, we demonstrate the associated effects on the video quality expressed in terms of the PSNR. Fig. 14 shows the PSNR video quality versus channel SNR for the AQAM modems using either $\mathrm{BCH}$ or turbo coding. The higher throughput of the $\mathrm{BCH}$ coded modem at high channel SNRs results in a slight advantage over the turbo coded modem in terms of PSNR. However, the reduced video packet loss ratio of the turbo coded modem below channel SNRs of $30 \mathrm{~dB}$ resulted in a higher effective throughput, increasing the video quality of the turbo coded modem. In conclusion, we have shown that the more complex turbo coded AQAM modem out performed the BCH-coded modem, since it reduced the video packet loss ratio and hence increased both the effective throughput and the video quality for all but high channel SNRs.

\section{CONCLUSION}

In contrast to previous statically reconfigured narrow-band multimode video transceivers [3]-[6] in this contribution we have proposed a wideband burst-by-burst adaptive video transceiver, which employs the channel SNR perceived by the channel equalizer as the quality measure for controlling the modem modes. Whilst in reference [22] the throughput upper-bound of such an AQAM modem was analyzed, in this contribution a practical video transceiver was introduced and the achievable video performance gains due to employing the proposed wideband burst-by-burst adaptive modem was quantified. When our adaptive packetizer is used in conjunction with the adaptive modem, it continually adjusts the video codec's target bitrate to match the instantaneous bitrate capacity provided by the adaptive modem.

We have also shown that a practical burst-by-burst adaptive modem exhibits a better performance than a statically configured multi-mode scheme. The delay between the channel estimation and modulation mode switching was shown to have a considerable effect on the performance achieved by the adaptive modem. This performance penalty can be mitigated by reducing the modem mode switching latency. However, at lower vehicular speeds the switching latency is less crucial, and the practical one-frame delay adaptive modem can achieve a performance that is closer to that of the ideal zero-delay adaptive modem which we used as an upper bound. We have also demonstrated how the transmission FER performance is affected by changing the switching thresholds. Therefore, the system can be tuned to the required FER performance using appropriate switching 
thresholds. Lastly, we have shown that-as expected-the more complex turbo channel codecs were more robust against channel effects than the lower-complexity binary $\mathrm{BCH}$ codecs.

\section{ACKNOWLEDGMENT}

The authors would like to thank the anonymous reviewers for constructive critiques.

\section{REFERENCES}

[1] L. Hanzo, W. T. Webb, and T. Keller, Single- and Multi-Carrier Quadrature Amplitude Modulation: Principles and Applications for Personal Communications, WATM and Broadcasting. New York: Wiley, Apr. 2000.

[2] L. Hanzo, P. Cherriman, and J. Streit. (Dec. 2000) Wireless Video Communications: Second to Third Generation Systems, WLAN's and Beyond. IEEE Press. [Online]. Available: http://www-mobile.ecs.soton.ac.uk

[3] J. Streit and L. Hanzo, "Vector-quantised low-rate cordless videophone systems," IEEE Trans. Vehic. Technol., vol. 42, pp. 340-357, May 1997.

[4] L. Hanzo and J. Streit, "Adaptive low-rate wireless videophone systems," IEEE Trans. Circuits Syst. Video Technol., vol. 5, pp. 305-319, Aug. 1995.

[5] J. Streit and L. Hanzo, "Quadtree-based reconfigurable cordless videophone systems," IEEE Trans. Circuits Syst. Video Technol., vol. 6, pp. 225-237, Apr. 1996.

[6] _ "Comparative study of programmable-rate videophone codecs for existing and future multimode wireless systems," Eur. Trans. Telecomm., vol. 8, no. 6, pp. 551-572, 1997.

[7] R. Steele and L. Hanzo, Eds., Mobile Radio Communications, 2nd ed. New York: Wiley, 1999

[8] Coding of Moving Pictures and Associated Audio for Digital Storage Media up to About 1.5 Mbit/s, Parts 1-3, ISO/IEC 11172 MPEG 1 International Standard, 1993.

[9] Information Technology, Generic Coding of Moving Video and Associated Audio Information, Parts 1-3, ISO/IEC CD 13818 MPEG 2 International Standard, 1995.

[10] ITU recommendation H.263: Video coding for low bitrate communication, ITU-T, 1996.

[11] P. Cherriman and L. Hanzo, "Programable H.263-based wireless video transceivers for interference-limited environments," IEEE Trans. Circuits Syst. Video Technol., vol. 8, pp. 275-286, June 1997.

[12] N. Faerber, E. Steinbach, and B. Girod, "Robust H.263 compatible transmission for mobile video server access," in Proc. 1st Int. Workshop on Wireless Image/Video Communications, Loughborough Univ., Loughborough, U.K., Sept. 4-5, 1996, pp. 8-13.

[13] A. H. Sadka, F. Eryurtlu, and A. M. Kondoz, "Improved performance H.263 under erroneous transmission conditions," Electron. Lett., vol. 33, no. 2 , pp. $122-124$, Jan. 16,1997

[14] W. T. Webb and R. Steele, "Variable rate QAM for mobile radio," IEEE Trans. Commun., vol. 43, pp. 2223-2230, July 1995.

[15] S. Sampei, S. Komaki, and N. Morinaga, "Adaptive modulation/TDMA scheme for large capacity personal multimedia communications systems," IEICE Trans. Commun., vol. E77-B, pp. 1096-1103, Sept. 1994.

[16] J. M. Torrance and L. Hanzo, "On the upper bound performance of adaptive QAM in a slow Rayleigh fading," Electron. Lett., pp. 169-171, April 1996.

[17] - , "Optimization of switching levels for adaptive modulation in a slow Rayleigh fading channel," Electron. Lett., pp. 1167-1169, June 1996.

[18] H. Matsuako, S. Sampei, N. Morinaga, and Y. Kamio, "Adaptive modulation systems with variable coding rate concatenated code for high quality multi-media communication systems," in Proc. IEEE Vehicular Technology Conf., 1996, pp. 487-491.

[19] S. G. Chua and A. Goldsmith, "Adaptive coded modulation for fading channels," in Proc IEEE Int. Conf. Communications, 1997, pp. $1488-1492$.
[20] A. J. Goldsmith and S. G. Chua, "Variable rate variable power MQAM for fading channels," IEEE Trans. Commun., vol. 45, pp. 1218-1230, Oct. 1997

[21] J. M. Torrance, "Adaptive full response digital modulation for wireless communications systems," Ph.D. dissertation, Univ. Southampton, Southampton, U.K., 1997.

[22] C. H. Wong and L. Hanzo, "Channel capacity upper-bound of a wideband burst-by-burst adaptive modem," in Proc. VTC'99, Houston, TX, May 1999, pp. 1851-1855.

[23] S. Otsuki, S. Sampei, and N. Morinaga, "Square-QAM adaptive modulation/TDMA/TDD systems using modulation estimation level with Walsh function," Electron. Lett., vol. 31, pp. 169-171, February 1995.

[24] J. M. Torrance and L. Hanzo, "Demodulation level selection in adaptive modulation," Electron. Lett., pp. 1751-1752, Sept. 1996.

[25] J. C. Cheung, "Adaptive equalisers for wideband TDMA mobile radio," $\mathrm{Ph} . D$. thesis, Univ. Southampton, Southampton, U.K., 1991.

[26] "COST 207: Digital land mobile radio communications, final report," Office for Official Publications of the European Communities, Luxembourg, 1989.

[27] A. Klein, R. Pirhonen, J. Skoeld, and R. Suoranta, "FRAMES multiple access mode 1 -wideband TDMA with and without spreading," in Proc. IEEE Int. Symp. Personal, Indoor and Mobile Radio Communications, PIMRC'97, vol. 1. Helsinki, Finland, Sept. 1-4, 1997, pp. 37-41.

[28] J. M. Torrance and L. Hanzo, "Latency and networking aspects of adaptive modems over slow indoors Rayleigh fading channels," IEEE Trans. Vehic. Technol., vol. 48, pp. 1237-1251, July 1999.

Peter Cherriman received the M.Eng. degree in information engineering in 1994 and the Ph.D. degree in mobile video networking, both from the University of Southampton, Southampton, U.K.

Since 1994, he has been with the Department of Electronics and Computer Science, University of Southampton. Currently, he is working on projects for the Mobile Virtual Centre of Excellence, Basingstoke, U.K. His current areas of research include robust video coding, microcellular radio systems, power control, dynamic channel allocation, and multiple access protocols. Further information and publications can be found at: http://www-mobile.ecs.soton.ac.uk.

Choong Hin Wong received the B.Eng. degree in electronics (with distinction) from the University of Southampton, Southampton, U.K., in 1995. During 1996-1999, he commenced postgraduate research in the Department of Electronics and Computer Science, University of Southampton, where he completed his thesis on burst-by-burst adaptive full-response multi-level modulation.

Lajos Hanzo received the Dipl.Ing. degree in electronics in 1976 and the Ph.D degree in 1983

During his 24-year career in telecommunications, he has held various research and academic posts in Hungary, Germany, and the U.K. Since 1986, he has been with the Department of Electronics and Computer Science, University of Southampton, Southampton, U.K., and has been a Consultant to Multiple Access Communications Ltd., U.K. He is currently Chair of Telecommunications. He has co-authored five books on mobile radio communications, coauthore over 300 research papers, organized and chaired conference sessions, presented overview lectures, and was awarded a number of distinctions. Currently, he is managing a research team, working on a range of research projects in the field of wireless multimedia communications under the auspices of the Engineering and Physical Sciences Research Council (EPSRC) U.K, the European IST Program, and the Mobile Virtual Center of Excellence (VCE). He also instructs a range of industrial training courses. For further information on research in progress and associated publications, please refer to http://www-mobile.ecs.soton.ac.uk. 